\title{
Comparative Study of Sag \& Swell Mitigation by a Novel Multi Level DVR with Wavelets
}

\author{
G. Devadasu \\ Department of EEE, CMR College of Engineering and Technology \\ Dr. M. Sushama \\ Department of EEE, JNTUH University Hyderabad, TS, India
}

\begin{abstract}
A Three Phase Dynamic Voltage Restorer (DVR) based on two, three \& five-level multilevel inverter topology has been proposed in this paper. Dynamic Voltage Restorers (DVRs) are a cost-effective solution to protect sensitive loads against voltage sags in medium- and high-power applications because the power required to compensate sags \& swell is only a fraction of the load rated power. Also, the energy storage requirements of multi level DVRs can be greatly reduced by using minimum-power compensation of voltage sags \& swells. However, this compensation strategy can disturb sensitive loads and tends to saturate the injection transformer unless a slow phase rotation technique is used when a voltage sag \& swell is detected. The sag \& swell detection needs to be robust, but also fast to provide accurate results so that the multi level DVR can work under all possible situations. This paper proposes and studies in detail a sag \&swell mitigating algorithm for a multi level DVR that, when designed and implemented carefully, provides excellent performance when the grid voltage contains harmonics and the grid frequency varies. This can verified by using THD analysis \& wavelets.
\end{abstract}

Keywords- sag, swell, Mitigation, DVR and THD

\section{INTRODUCTION}

Modern domestic and industrial equipments are extremely sensitive to voltage changes. As a result, maintenance of power quality in the distribution power network becomes an important issue. The quality of the power is mainly determined by voltage magnitude, frequency and its wave-shape. In a distributed power system network, voltage disturbances are the main causes for power quality issues. Voltage sag/swell, voltage flicker, harmonic voltage component, voltage unbalance are mainly responsible for voltage disturbance. Of all these, voltage sag is the most dominant power quality problem. It has a major impact on sensitive loads. The cost of interruption of an industrial process due to voltage disturbance might be larger than the severity of the causes. This is actually the main reason for the development of Dynamic Voltage Restorer. If the line voltage reduces due to sag or increases due to swell, then the DVR injects voltage according to the situation to maintain the load voltage at desired value. Dynamic voltage restorer consists of DC storage device, voltage source inverter (VSI), passive filter components and voltage injection transformer. A appropriate control strategy is required to control the switches of inverter to synthesize desired voltage. Different topologies of DVR have been proposed in the literature. It is observed that two-level H-bridge inverters are mainly used for this purpose. On the other hand, multilevel inverter has better harmonic characteristics (better THD) and less filter requirements to eliminate the switching harmonics. There are different multilevel inverter topologies available in the literature.

\section{MULTILEVEL CONVERTER}

A few well known types of multilevel inverters are

(1) Cascade H-bridge multilevel inverter

(2) Flying capacitor multilevel inverter

(3) Diode-clamped multilevel inverter etc. 
An inverter is an electrical device that converts direct current (DC) to alternating current (AC); the converted AC can be at any required voltage and frequency with the use of appropriate transformers, switching, and control circuits.

Static inverters have no moving parts and are used in a wide range of applications, from small switching power supplies in computers, to large electric utility high-voltage direct current applications that transport bulk power. Inverters are commonly used to supply AC power from DC sources such as solar panels or batteries.

The electrical inverter is a high-power electronic oscillator. It is so named because early mechanical AC to DC converters was made to work in reverse, and thus were "inverted", to convert DC to AC.

The inverter performs the opposite function of a rectifier.

CASCADED H-BRIDGES INVERTER:A single-phase structure of an m-level cascaded inverter is illustrated in Figure 1. Each separate dc source (SDCS) is connected to a single-phase full-bridge, or H-bridge, inverter. Each inverter level can generate three different voltage outputs, $+\mathrm{V}_{\mathrm{dc}}, 0$, and $-\mathrm{V}_{\mathrm{dc}}$ by connecting the dc source to the ac output by different combinations of the four switches, $S_{1}, S_{2}, S_{3}$, and $S_{4}$. To obtain $+V_{d c}$, switches $S_{1}$ and $S_{4}$ are turned on, whereas $-V_{d c}$ can be obtained by turning on switches $S_{2}$ and $S_{3}$. By turning on $S_{1}$ and $S_{2}$ or $S_{3}$ and $S_{4}$, the output voltage is 0 . The ac outputs of each of the different full-bridge inverter levels are connected in series such that the synthesized voltage waveform is the sum of the inverter outputs.

The number of output phase voltage levels $m$ in a cascade inverter is defined by $m=2 s+1$, where $s$ is the number of separate dc sources. An example phase voltage waveform for an 11-level cascaded $\mathrm{H}$-bridge inverter with 5 SDCSs and 5 full bridges is shown in Figure 1 The phase voltage $v_{a n}=v_{a 1}+v_{a 2}+v_{a 3}+v_{a 4}+v_{a 5}$.

For a stepped waveform such as the one depicted in Figure 31.2 with s steps, the Fourier Transform for this waveform follows

$$
V(\omega t)=\frac{4 V_{d c}}{\pi} \sum_{n}\left[\cos \left(n \theta_{1}\right)+\cos \left(n \theta_{2}\right)+\ldots+\cos \left(n \theta_{s}\right)\right] \frac{\sin (n \omega t)}{n}, \text { where } n=1,3,5,7, \ldots
$$




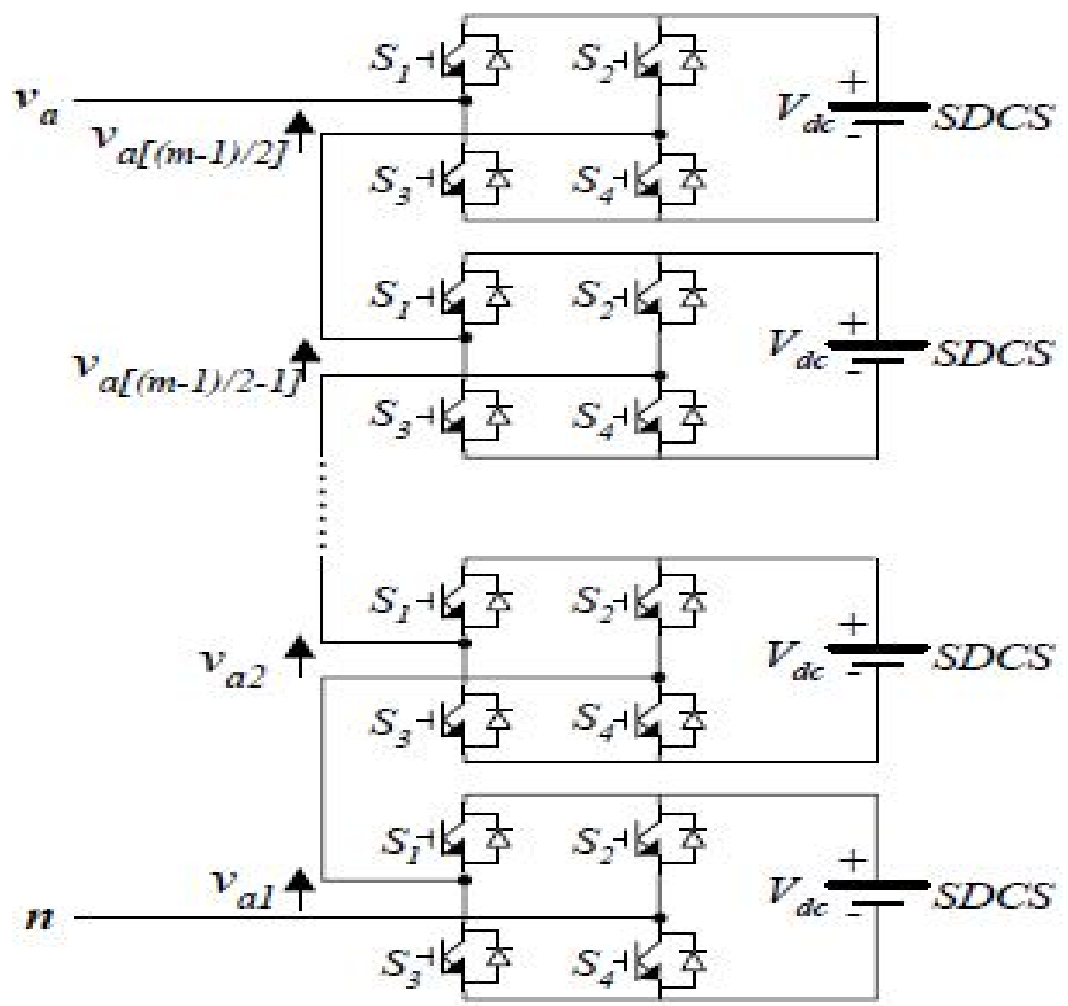

Fig 1: Single-phase structure of a multilevel cascaded H-bridges inverter 


\section{SIMULATION DESIGN OF MULTI LEVEL DVR}

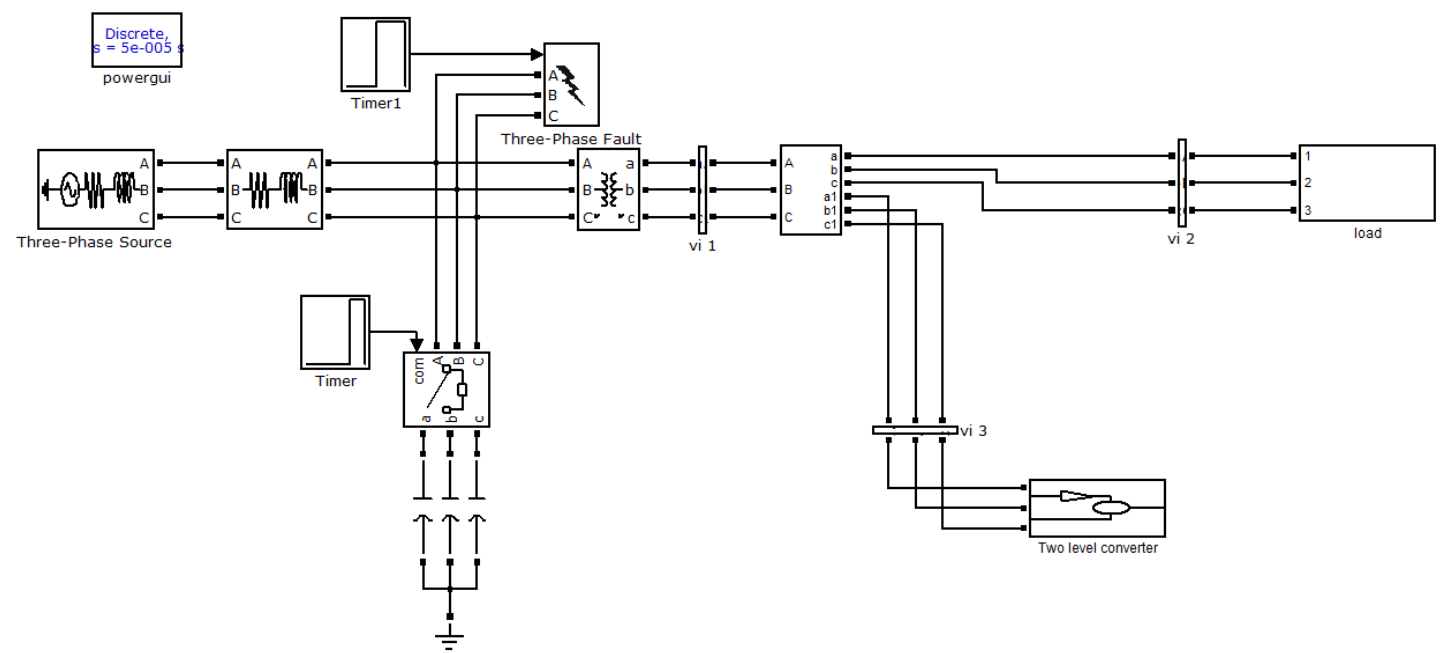

Fig 2: matlab simulation ckt of multi level DVR

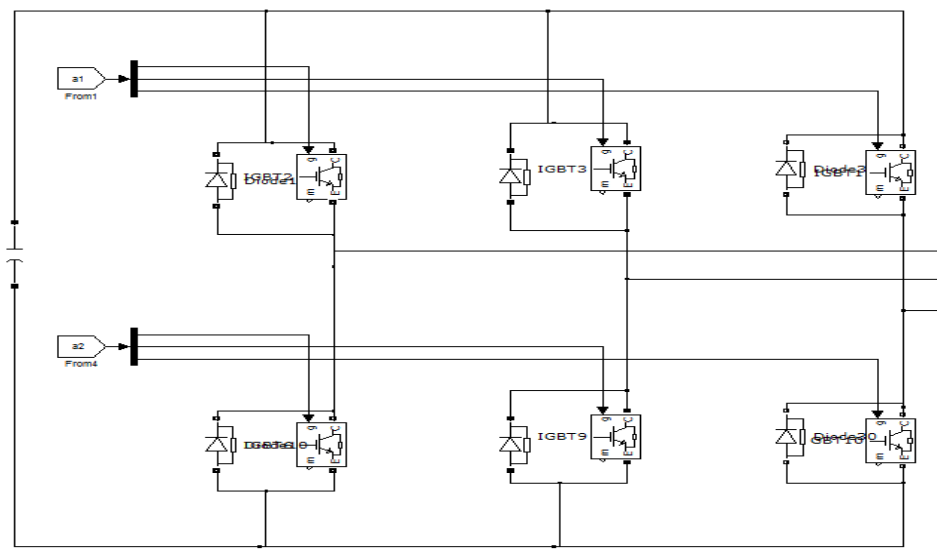

Fig 3: two level converter based DVR system 

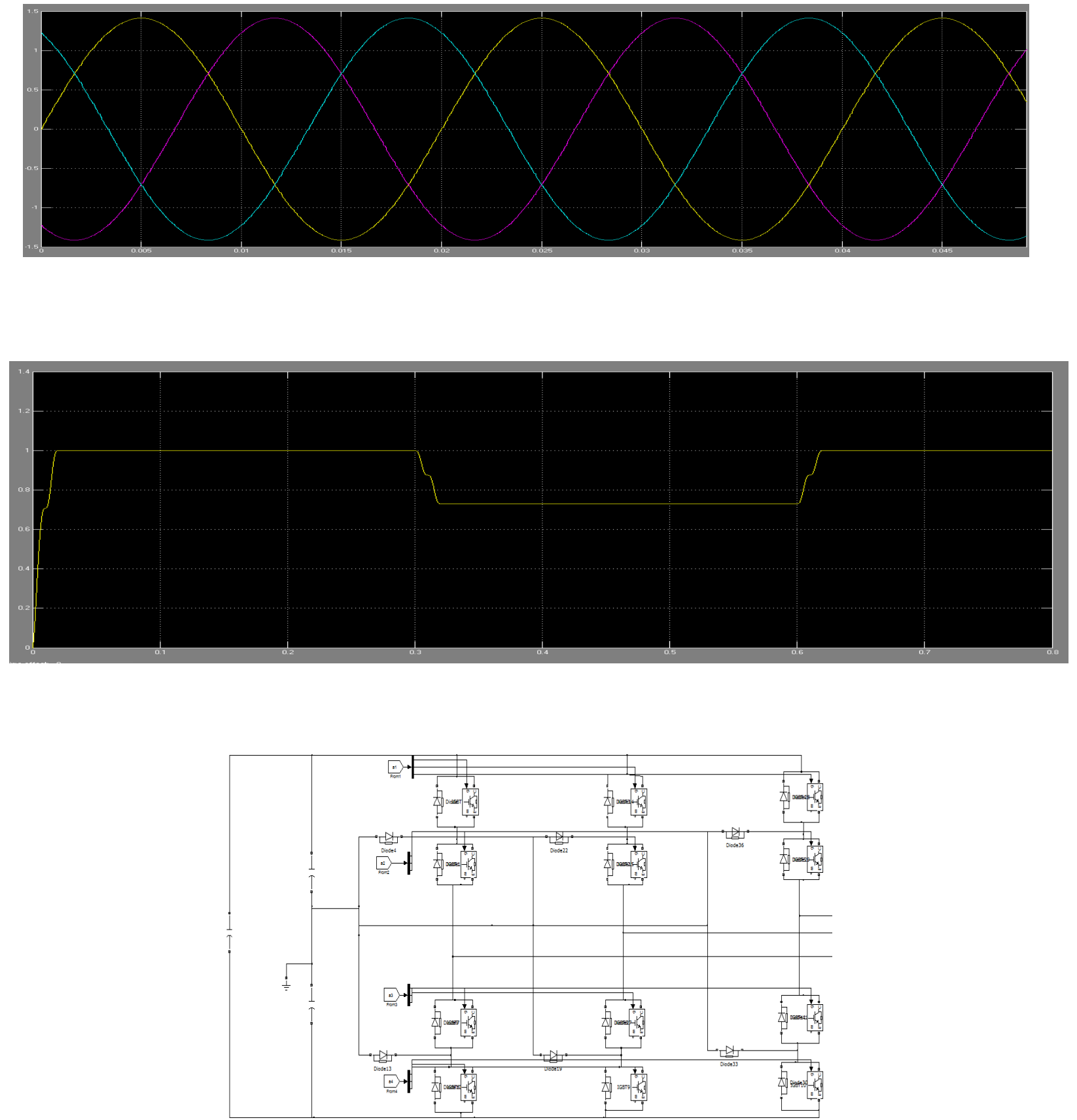

Fig 3: three level converter based DVR system 

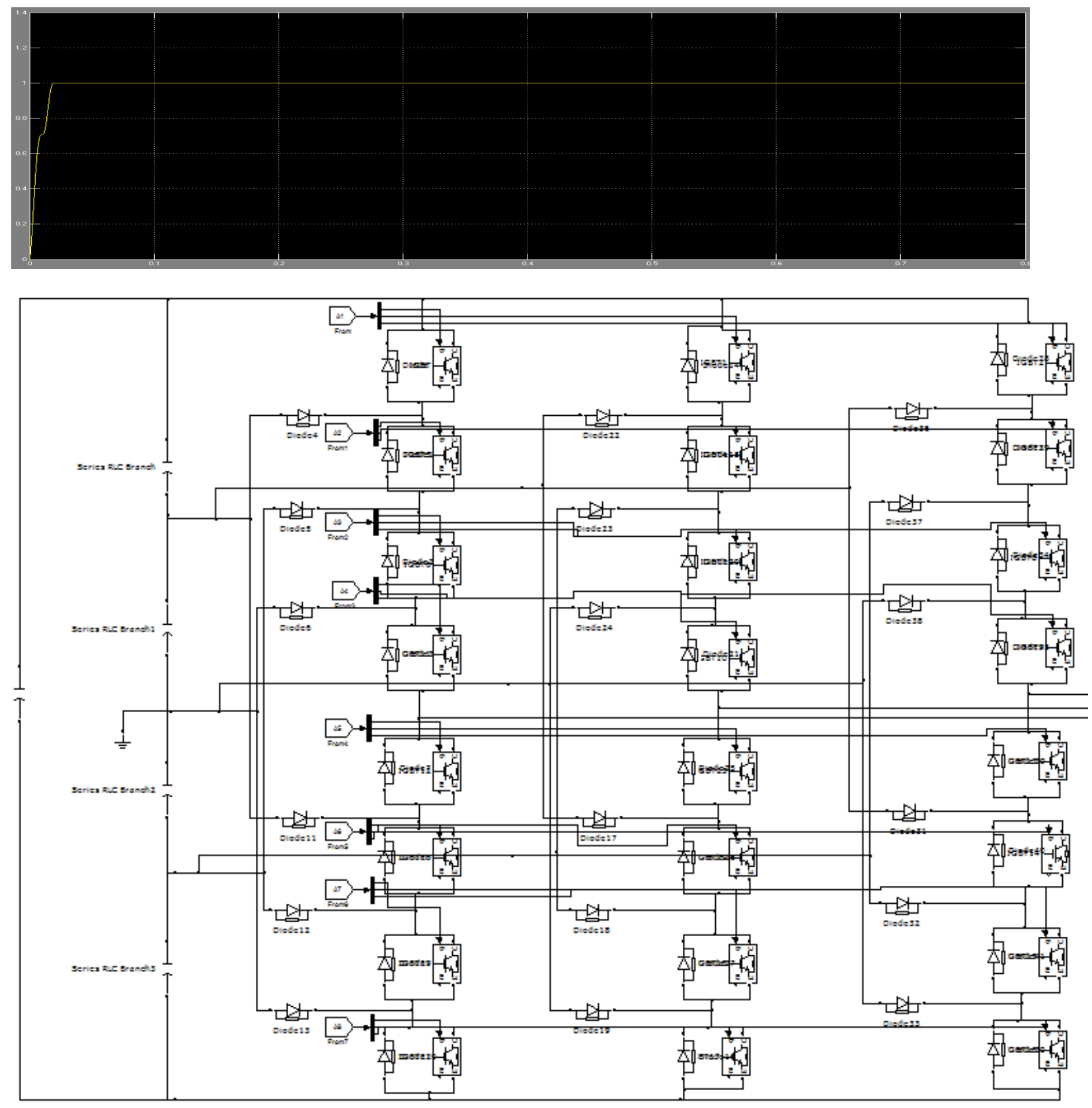

Fig 5: five level converter based DVR system 


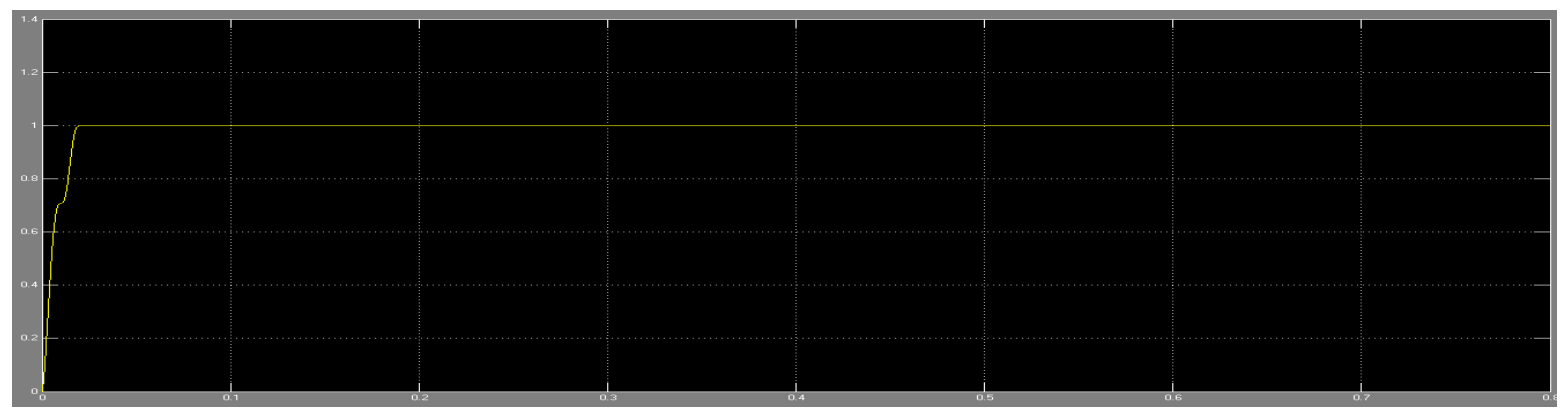

\section{CONCLUSION}

In this paper an overview of multi level DVR is presented. DVR is an effective custom power device for voltage sags and swells mitigation. The impact of voltage sags on sensitive equipment is severe. Therefore, DVR is considered to be an efficient solution due to its relatively low cost and small size, also it has a fast dynamic response. The simulation results show clearly the performance of a multi level DVR in mitigating voltage sags and swells. The multi level DVR handles both balanced and unbalanced situations without any difficulties and injects the appropriate voltage component to correct rapidly any anomaly in the supply voltage to keep the load voltage balanced and constant at the nominal value.

\section{REFERENCES}

[1] IEEE Guide for Voltage Sag Indices," in IEEE Std 1564-2014, vol., no., pp.1-59, June 202014

[2] IEEE Guide for Identifying and Improving Voltage Quality in Power Systems," in IEEE Std 1250-2011 (Revision of IEEE Std 1250-1995), vol., no., pp.1-70, March 312011

[3] A. Ghosh and G. Ledwich, "Structures and control of a dynamic voltage regulator (DVR)," Power Engineering Society Winter Meeting, 2001. IEEE, Columbus, OH, 2001, pp. 1027-1032 vol.3. doi: 10.1109/PESW.2001.917209

[4] Huiwen Liu, Bowen Zheng and Xiong Zhan, "A comparison of two types of storageless DVR with a passive shunt converter," 2016 IEEE 8th International Power Electronics and Motion Control Conference (IPEMC-ECCE Asia), Hefei, 2016, pp. 1280-1284.

[5] P. C. Loh, D. M. Vilathgamuwa, S. K. tang, H. L. Long, ”Multilevel dynamic voltage restorer", IEEE Power Electronic Letters, vol. 2, no. 4, pp. 125-130, Dec. 2004.

[6] A. Khoshkbar Sadigh, E. Babaei, S. H. Hosseini and M. Farasat "Dynamic voltage restorer based on stacked multicell converter," IEEE International Symposium on Industrial Electronics and Application (ISIEA2009), Malaysia, pp.419424.

[7] A. Delavari, I. Kamwa and A. Zabihinejad, "A comparative study of different multilevel converter topologies for high power photovoltaic applications," 2016 7th Power Electronics and Drive Systems Technologies Conference (PEDSTC), Tehran, 2016, pp. 415-420 